\title{
Molecular basis for the substrate stereoselectivity in Tryptophan Dioxygenase
}

\author{
Luciana Capece $^{1}$, Ariel Lewis-Ballester ${ }^{2}$, Marcelo A. Marti ${ }^{1,3}$, Dario A. Estrin ${ }^{1,{ }^{*}}$, and Syun- \\ Ru Yeh ${ }^{2, *}$ \\ ${ }^{1}$ Departamento de Química Inorgánica, Analítica y Química Física/INQUIMAE-CONICET \\ Facultad de Ciencias Exactas y Naturales, Universidad de Buenos Aires, Ciudad Universitaria, \\ Pabellón 2, Buenos Aires, C1428EHA, Argentina \\ ${ }^{2}$ Department of Physiology and Biophysics, Albert Einstein College of Medicine, 1300 Morris Park \\ Avenue, Bronx, New York 10461 USA \\ ${ }^{3}$ Departamento de Química Biológica, Facultad de Ciencias Exactas y Naturales, Universidad de \\ Buenos Aires, Ciudad Universitaria, Pabellón 2, Buenos Aires, C1428EHA, Argentina
}

\begin{abstract}
Tryptophan dioxygenase (TDO) and Indoleamine 2,3 dioxygenase (IDO) are the only two hemeproteins that catalyze the oxidation reaction of tryptophan (Trp) to N-formylkynurenine (NFK). While human IDO (hIDO) is able to oxidize both L and D-Trp, human TDO (hTDO) displays a major specificity towards L-Trp. In this work we aim to interrogate the molecular basis for the substrate stereoselectivity of hTDO. Our previous molecular dynamics simulation studies of Xanthomonas campestris TDO (xcTDO) showed that an H-bond between T254 (T342 in hTDO) and the ammonium group of the substrate is present in the L-Trp-bound enzyme, but not in the DTrp bound enzyme. The fact that this is the only notable structural alteration induced by the change in the stereo structure of the substrate prompted us to produce and characterize the T342A mutant of hTDO to evaluate the structural role of T342 in controlling the substrate stereoselectivity of the enzyme. The experimental results indicate that the mutation only slightly perturbs the global structural properties of the enzyme, but it totally abolishes the substrate stereoselectivity. Molecular Dynamics simulations of xcTDO show that T254 controls the substrate stereoselectivity of the enzyme by (i) modulating the H-bonding interaction between the $\mathrm{NH}_{3}{ }^{+}$group and epoxide oxygen of the ferryl/indole 2,3-epoxide intermediate of the enzyme, and (ii) regulating the dynamics of two active site loops, loop $_{250-260}$ and loop $117-130$, critical for substrate-binding.
\end{abstract}

Tryptophan dioxygenase (TDO) is a heme-containing enzyme that catalyzes the conversion of L-Tryptophan (L-Trp) to N-Formyl kynurenine (NFK), which represents the first and rate-limiting step of the L-Trp catabolism through the kynurenine pathway. ${ }^{1}$ TDO is a ubiquitous enzyme found in bacteria, insects and mammals. In mammals, it is expressed mainly in the liver, where it is responsible for L-Trp processing that ultimately leads to the biosynthesis of NAD and NADP. ${ }^{2,3}$.

\footnotetext{
*To whom correspondence should be addressed: Dario A. Estrin: dario@qi.fcen.uba.ar. Phone: +541145763380. Fax:+541145763341. Syun-Ru Yeh: syun-ru.yeh@einstein.yu.edu. Phone: 718-430-4234. Fax:718-430-4230.

Supplementary Information. Sequence alignment of various isoforms of TDO, SDS-PAGE data of the T324 mutant, kinetic trances for the product formation from the T254A mutant, Resonance-Raman spectra for the Trp-CO complexes of xcTDO, RMSD and Potential energy of the Trp- $\mathrm{O}_{2}$ complexes and ferryl/L-indole 2,3-epoxide intermediates of xcTDO as a function of the MD simulation time, and calculated work required for the transition between the closed and open conformations of the ferryl/L-indole 2,3-epoxide intermediate of xcTDO are available free of charge via the Internet at http://pubs.acs.org.
} 
Indoleamine 2,3-dioxygenase (IDO) is also a heme-containing enzyme that catalyzes the same oxidation reaction of L-Trp. Contrary to TDO, in mammals IDO is expressed in all tissues other than the liver. Recently, significant efforts have been put forth to unravel the reaction and inhibition mechanisms of IDO and TDO, as promoted by the discovery that IDO plays a pivotal role in cancer immune escape. ${ }^{4,5,67,8}$ Along this line, it has been demonstrated that the combination of a IDO inhibitor, 1-methyl Trp, and cytotoxic chemotherapy leads to significant tumour regression in mouse model systems. ${ }^{4}$ In this context, it is important to develop potent IDO inhibitors that do not interfere with normal TDO function.

The crystal structure of mammalian TDO is not known; nonetheless those of two bacterial isoforms of TDO, from Xanthomonas campestris (xcTDO, PDB code: $2 \mathrm{NW} 8$ ) $^{9}$ and Ralstonia metallidurans (rmTDO, PDB code: $2 \mathrm{NOX}$ ) ${ }^{10}$ have been published in 2007. In the case of xcTDO, the enzyme was crystallized in both substrate-free and L-Trp-bound forms. In the structure of the L-Trp bound form, several tight contacts between L-Trp and the enzyme are evident (Fig. 1), including H-bonding interactions between the carboxylate group of L-Trp and Y113/R117, the ammonium group of L-Trp, T254 and the propionate A group of the heme, and the indoleamine group of L-Trp and H55, as well as the hydrophobic interactions between L-Trp, F51, L120 and F116 (it is noted that F116 is not shown in Fig. 1 for clarity). Structure-based sequence alignment data suggest that most of the key interactions between the substrate and the enzyme observed in XcTDO are conserved in human IDO (hIDO). ${ }^{9}, 11,12$ Consistently, our hybrid Quantum Mechanics and Molecular Mechanics (QM-MM) studies ${ }^{13,14}$ showed that XcTDO and hIDO carry out the Trp dioxygenation reaction with a similar ferryl-based mechanism. Based on this mechanism, the heme iron bound dioxygen is first inserted into the $\mathrm{C}_{2}$ atom of Trp to generate a ferryl/ indole 2,3-epoxide intermediate. The subsequent ring opening reaction of the epoxide, catalyzed by a proton transfer from the ammonium group to the epoxide, triggers the insertion of the ferryl oxygen to the $\mathrm{C}_{2}$ atom to generate the NFK product. In this scenario, the proper positioning of the ammonium group of the substrate, as well as the electronic environment surrounding it, are believed to be critical for the catalysis.

Although TDO and IDO exhibits high structural similarity and follow a similar dioxygenase mechanism, they exhibit several intriguing differences in the substrate stereoselectivity: hTDO shows comparable affinities towards L-Trp and D-Trp, but the $k_{\text {cat }}$ for L-Trp is 10fold higher with respect to D-Trp; ${ }^{12}$ in contrast, hIDO displays $\sim 170$-fold higher affinity towards L-Trp with respect to D-Trp ${ }^{15}$, but the $k_{\text {cat }}$ are similar for the two stereoisomers. The structural factors leading to the differences in the substrate stereoselectivity of the two enzymes are unclear. Nonetheless, our previous molecular dynamics (MD) simulation data ${ }^{16}$ suggest that most of the interactions between the substrate and the enzyme in xcTDO are conserved in the L-Trp and D-Trp-bound complexes, except that the strong H-bond between the ammonium group of L-Trp and the OH group of T254 (equivalent to T342 in hTDO) is significantly weakened in D-Trp-bound enzyme, implying that T254 plays a critical role in controlling the substrate stereoselectivity of xcTDO.

To define the role of T342 in the substrate stereoselectivity of hTDO, we have produced and characterized the T342A mutant by using resonance Raman and optical absorption spectroscopies, and determined its activity towards L-Trp and D-Trp. Our data show that the T342A mutation only causes minor changes to the global structure of the enzyme, but it totally abolishes the substrate stereoselectivity. To unveil the structural perturbations to the enzyme underlying its absence of substrate stereoselectivity, the experimental work was complemented with classical MD simulations. As the crystal structure of hTDO is not available, the simulations were carried out with xcTDO. The simulation data of the L- and D-Trp bound $\mathrm{O}_{2}$ complexes, as well as the corresponding ferryl/indole 2,3-epoxide 
intermediates, indicate that T254 controls the substrate stereoselectivity of xcTDO by modulating the H-bonding interaction between the ammonium group and epoxide oxygen of the ferryl/indole 2,3-epoxide intermediate, and by regulating the interactions between two critical loops, loop $250-260$ and loop $117-130$, that sequester substrate in the active site.

\section{Materials and Methods}

\section{Expression and Purification of the wild type hTDO}

The details of protein expression and purification are described elsewhere. ${ }^{12,}{ }^{17}$ Briefly, the hTDO protein, with $\mathrm{N}$ - and C-terminal tails truncated, plus a $6 \mathrm{X}$-His tag extension at the $\mathrm{C}$ terminus, was over-expressed in E. coli BL21 Star (DE3) cells by using the pET30b vector (Stratagene, La Jolla, CA). The transformant was selected from a single colony on a LB kanamycin agar plate and was used to inoculate a LB medium, supplemented with $50 \mu \mathrm{g} / \mathrm{mL}$ kanamycin. This starter culture was grown overnight at $37^{\circ} \mathrm{C}$, and was subsequently used to inoculate a $1 \mathrm{~L}$ of $\mathrm{LB}$ medium. The resulting $E$. coli culture was grown at $37^{\circ} \mathrm{C}$ in a shaker at $250 \mathrm{rpm}$ until the optical density at $600 \mathrm{~nm}$ reached $\sim 0.8$. The expression of hTDO was induced by Isopropyl $\beta$-D-1-thiogalactopyranoside (IPTG), with a final concentration of 1 $\mathrm{mM}$. An aliquot of hemin, with a final concentration of $8-10 \mu \mathrm{M}$, was added to the culture to ensure the complete incorporation of the heme prosthetic group into the recombinant protein. The culture was grown at $25^{\circ} \mathrm{C}$ for additional $6-8 \mathrm{hrs}$ in a shaker at $150 \mathrm{rpm}$. The cells were harvested by centrifugation and stored at $-20{ }^{\circ} \mathrm{C}$ until use.

The recombinant protein was purified by affinity chromatography with a Ni-NTA column (Novagen). The protein was eluted with $250 \mathrm{mM}$ imidazole (Sigma) in $50 \mathrm{mM}$ phosphate buffer (pH 7.8) and $250 \mathrm{mM} \mathrm{KCl}$. To stabilize the protein, $10 \mathrm{mM} \mathrm{L-Trp}$ and 5\% glycerol was present throughout the purification procedure. To ensure that the protein was in its ferric state, it was oxidized with potassium ferricyanide, and subsequently passed through a sephadex G25 column to remove the ferricyanide, L-Trp, imidazol and glycerol. The purity of the protein was confirmed by SDS PAGE analysis as shown in Fig. S2. The protein thus collected was stored in $100 \mathrm{mM}$ phosphate buffer $(\mathrm{pH} 7.5)$ with $150 \mathrm{mM} \mathrm{KCl}$ at $-80^{\circ} \mathrm{C}$ until use.

The T342A mutant was created by using the QuikChange II kit (Stratagene) with the following primers: sense primer: 5'-CAGCAAAGCTGGCGCCGGTGGTTCCTC-3' and antisense primer: 5'-GAGGAACCACCGGCGCCAGCTTTGCTG-3'. The sequence was verified by DNA sequence analysis. The mutant was cloned, expressed, and purified by using the same protocol as that described for the wild type protein.

\section{Steady-State Activity Assay}

For the activity assay, the ferric hTDO was rapidly mixed with sodium ascorbate (100-fold excess with respect to the protein) in the presence of a desired amount of L-Trp in $\mathrm{pH} 7.0$ phosphate buffer $(100 \mathrm{mM})$. The temperature was controlled with a water bath at $25^{\circ} \mathrm{C}$. The final concentration of hTDO was $0.5 \mu \mathrm{M}$. The reaction rate was followed by monitoring the product formation at $321 \mathrm{~nm}\left(\varepsilon=3750 \mathrm{M}^{-1} \mathrm{~cm}^{-1}\right.$ for $N$-formyl kynurenine $) .{ }^{12,18}$ Two representative kinetic traces obtained with $8 \mathrm{mM} \mathrm{L-}$ and D-Trp are shown in Fig. S3. The initial product formation rate was plotted as a function of L-Trp concentration; the data were analyzed by Michealis-Menten curve fitting with Origin 6.1 software (Microcal Software, Inc., MA).

\section{Spectroscopic Measurements}

The optical absorption spectra were taken on a spectrophotometer, UV2100, from Shimadzu Scientific Instruments, Inc. (Columbia, MD) with a spectral slit width of $1 \mathrm{~nm}$. The 
resonance Raman spectra were taken on the instrument described elsewhere. ${ }^{19}$ Briefly, the $413.1 \mathrm{~nm}$ excitation from a Kr ion laser (Spectra Physics, Mountain View, CA) was focused to a $30 \mu \mathrm{m}$ spot on a spinning quartz cell rotating at $1000 \mathrm{rpm}$. The scattered light, collected at a right angle to the incident laser beam, was focused on the $100 \mu \mathrm{m}$-wide entrance slit of a $1.25 \mathrm{~m}$ Spex spectrometer equipped with a 1200 grooves/mm grating (Horiba Jobin Yvon, Edison, NJ), where it was dispersed and then detected by a liquid nitrogen-cooled CCD detector (Princeton Instruments, Trenton, NJ). A holographic notch filter (Kaiser, Ann Arbor, MI) was used to remove the laser line. The Raman shift was calibrated by using indene (Sigma) as a reference. The laser power was kept at $\sim 5 \mathrm{~mW}$ for all measurements, except that used for the $\mathrm{CO}$ complexes, in which it was kept at $<1 \mathrm{~mW}$ to avoid photodissociation of the heme-bound $\mathrm{CO}$. The acquisition time was 30 min for all except that of the super high frequency spectra of the CO-bound complexes in which the acquisition time was $180 \mathrm{~min}$. The ferrous derivative was prepared by reducing the ferric protein, prepurged with $\mathrm{N}_{2}$ gas, with sodium dithionite under anaerobic conditions. The CObound ferrous complexes were obtained by gentle purging $\mathrm{CO}$ gas on the surface of the solution containing the ferrous form of the enzyme under anaerobic conditions. The concentration of the protein samples used for the Raman measurements was $50 \mu \mathrm{M}$ in $\mathrm{pH}$ 7.0 phosphate buffer $(100 \mathrm{mM})$.

\section{Starting structures for classical Molecular Dynamics Simulations}

Due to the fact that the crystal structure of hTDO is not available, we have performed the simulations on the XcTDO structure. Sequence comparison of hTDO and XcTDO with 7 other TDOs from various organisms (Fig. S1) indicates that most of the residues involved in Trp binding are conserved, including H76 (55 in xcTDO), R144 (117 in xcTDO), F72 (51 in xcTDO), F143 (116 in xcTDO), L147 (120 in xcTDO), with the exception of Y113 of xcTDO that is replaced by a Phe residue in all the analyzed mammalian and insect sequences. Additionally, the $\operatorname{loop}_{250-260}$ and loop ${ }_{117-130}$ involved in the observed conformational change in the T254A mutant of xcTDO display high sequence homology, with all relevant residues involved in the interactions conserved. In this context, xcTDO structure provides a good model of the human protein justifying its use for the MD simulations.

The structure of the ternary complex of xcTDO was obtained based on the crystal structure of the ferrous L-Trp-bound xcTDO (PDB code: 2NW8) ${ }^{9}$, as described eleswhere. ${ }^{13,16}$ Briefly, a one-subunit model of xcTDO was constructed by removing residues E19 to S35 from subunit A, and adding residues R21 to S35 of subunit B, which corresponds to a short helix that penetrates into the structure of the A subunit. The structure of D-Trp-bound xcTDO was obtained by a docking protocol described in reference. ${ }^{16}$ The structure of the ferryl/L or D-indole 2,3-epoxide intermediate of xcTDO was obtained from the QM-MM simulations of the corresponding ternary complexes. ${ }^{13,20}$ Starting from the structures of the ternary complexes and the ferryl/indole 2,3-epoxide intermediates of the wild type enzyme, we constructed the corresponding T254A mutant derivatives in-silico. These structures were used for all the MD simulations.

\section{Classical Molecular Dynamics Simulations}

The starting structure for each complex, was immersed in a pre-equilibrated octahedral box of TIP3P water molecules. The standard protonation state at physiological $\mathrm{pH}$ was assigned to ionizable residues. Special attention was paid to the protonation states of histidines, which were assigned on the basis of the H-bonding patterns with neighboring residues. All simulations were performed at $300 \mathrm{~K}$ and pressure of 1 bar using Berendsen thermostat and barostat ${ }^{21}$. Periodic boundary conditions and Ewald sums (grid spacing of $1 \AA$ ) were used to treat long range electrostatic interactions. The SHAKE algorithm was used to keep bonds 
involving hydrogen atoms at their equilibrium length. A 2 fs time step was used for the integration of Newton's equations. The Amber ff99SB force field ${ }^{22}$ was used for all residues but the heme, whose parameters were developed and thoroughly tested by our group in previous works ${ }^{23}, 24$. The parameters for the ferryl/indole 2,3-epoxide intermediate were obtained using standard Amber procedures, using ab-initio HF calculations and RESP ${ }^{22}$ method for determining the partial charges. All simulations were performed with the PMEMD module of the AMBER9 package. ${ }^{25}$ Equilibration consisted of an energy minimization of the initial structures, followed by a slow heating up to $300 \mathrm{~K}$. For each structure 20ns MD production runs were performed. Frames were collected at 2ps intervals, which were subsequently used to analyse the trajectories. Finally, in order to estimate the work required to produce the rotation of the $\mathrm{C}_{\alpha}-\mathrm{C}_{\beta}$ bond in the ferryl/indole 2,3-epoxide intermediate of the wild type and T254A mutant of xcTDO and make a rough estimation of the free energy profile for the inter-conversion of the two extreme structures, we added a time-dependent potential that moves the system from the initial to the final configuration, resulting $E^{\prime}(r)=E(r)+k\left[\left(\xi-\xi_{o}\right)^{2}\right]^{26}$. The constant $k$ was $200 \mathrm{kcal} / \mathrm{mol}$. $\mathrm{K}$ in all cases, and the coordinate $\xi$ was chosen as the dihedral angle formed by the $\mathrm{N}-\mathrm{C}_{\alpha}-\mathrm{C}_{\beta}-\mathrm{C}_{\gamma}$ atoms of the epoxide adduct (Fig. S9).

\section{Results and Discussion}

\section{Optical absorption and resonance Raman spectra of the T342A mutant of hTDO}

Fig. 2 shows the optical absorption spectra of the T342A mutant in the absence and presence of L-Trp. In the substrate-free form, the spectra of the ferric T342A shows a Soret band at $404 \mathrm{~nm}$, and a charge transfer band at $635 \mathrm{~nm}$, characteristic for a six-coordinate (6C) waterbound ferric heme, similarly to that reported for the wild type enzyme. ${ }^{12}$ The ferrous derivative has a Soret band at $427 \mathrm{~nm}$, and a visible band at $556 \mathrm{~nm}$, consistent with a fivecoordinate $(5 \mathrm{C})$ high-spin heme. The CO-bound complex, on the other hand, shows a Soret band at $419 \mathrm{~nm}$, and two visible bands at 539 and $565 \mathrm{~nm}$, confirming the presence of a $6 \mathrm{C}$ low-spin heme. Like the wild type enzyme, the addition of L-Trp or D-Trp to the mutant does not introduce significant modifications to the spectra, as summarized in Table 1.

The resonance Raman spectra of the ferric derivatives of the T342A mutant are shown in Fig. 3A. The substrate-free enzyme has a $v_{4}$ mode at $1372 \mathrm{~cm}^{-1}$, and $v_{2} / v_{3}$ modes at $1560 / 1482$ and $1581 / 1507 \mathrm{~cm}^{-1}$, indicating a $6 \mathrm{C}$ high-spin/low-spin mixed configuration, in good agreement with a water-bound ferric heme, as indicated by the optical absorption spectral data. The addition of L-Trp or D-Trp to the ferric enzyme does not perturb the spectral feature, except that the low-spin component is slightly increased, similar to that observed in the wild type enzyme (Fig. 3B).

The ferrous derivative of the T342A mutant exhibits $v_{3}$ and $v_{4}$ modes at 1470 and 1355 $\mathrm{cm}^{-1}$, respectively, in the absence of substrate, indicating a $5 \mathrm{C}$ high-spin heme (data not shown), same as the reported data for the wild type enzyme. ${ }^{12}$ In the low frequency window of the spectrum, the Fe-His stretching mode $\left(v_{\mathrm{Fe}-\mathrm{His}}\right)$ is identified at $227 \mathrm{~cm}^{-1}$ (Fig. 4A), similar to that of the wild type enzyme (Fig. 4B and Table 1), ${ }^{12}$ indicating that the mutation does not perturb the proximal heme environment. Nonetheless, the relative intensities of several in-plane and out-of-plane heme modes in the $250-450 \mathrm{~cm}^{-1}$ and $700-800 \mathrm{~cm}^{-1}$ region of the spectrum ${ }^{17,20}$ are significantly perturbed by the mutation, suggesting conformational changes to the heme prosthetic group due to the mutation. L-Trp or D-Trp binding to the mutant does not introduce noticeable changes to the spectrum, in contrast to the small changes observed in the wild type enzyme (Fig. 4B), manifesting slightly weaker substrate-enzyme interactions in the mutant enzyme. 
$\mathrm{CO}$ has been demonstrated to be a useful probe for investigating active site structure of heme-proteins. The spectral features of the CO-complex of T342A mutant (Fig. S4) are similar to those reported for the wild type enzyme, ${ }^{12}$ and are not affected by the addition of L-Trp or D-Trp, expect that the Fe-CO stretching mode $\left(v_{\mathrm{Fe}-\mathrm{CO}}\right)$ at $495 \mathrm{~cm}^{-1}$ found in the substrate-free enzyme shifted to $488 \mathrm{~cm}^{-1}$ in responding to L-Trp or D-Trp binding. The assignments of the $v_{\mathrm{Fe}-\mathrm{CO}}$ modes at 495 and $488 \mathrm{~cm}^{-1}$, along with those of the associated C$\mathrm{O}$ stretching modes $\left(v_{\mathrm{C}-\mathrm{O}}\right)$ at 1963 and $1970 \mathrm{~cm}^{-1}$, were confirmed by ${ }^{12} \mathrm{CO}-{ }^{13} \mathrm{CO}$ isotope substitution experiments (insert in Fig. S4). As listed in Table 1, in general, all the spectral features, as well as their responses to substrate binding, of the various derivatives of the T342A mutant are similar to those reported for the wild type enzyme, ${ }^{12}$ confirming that the mutation does not introduce major structural modifications to the enzyme.

\section{Activity of the T342A mutant of hTDO towards L and D-Trp}

To determine how the T342A mutation affects the enzyme activity, NFK production activities of the wild type and T342A mutant were followed as a function L-Trp or D-Trp concentration. As shown in Fig. 5B, the activity of the wild type enzyme towards either LTrp (black symbols) or D-Trp (red symbols) follows typical Michaelis-Menten behaviour with $k_{\text {cat }} / \mathrm{K}_{\mathrm{m}}$ of $2.24 \pm 0.08 \mathrm{~s}^{-1} / 0.12 \pm 0.02 \mathrm{mM}$ and $0.20 \pm 0.06 \mathrm{~s}^{-1} / 0.26 \pm 0.02 \mathrm{mM}$, respectively. The $k_{\text {cat }} / \mathrm{K}_{\mathrm{m}}$ values are similar to those reported previously $\left(2.1 \mathrm{~s}^{-1} / 0.19 \mathrm{mM}\right.$ and $0.2 \mathrm{~s}^{-1} / 0.18 \mathrm{mM}$ for L- and D-Trp, respectively); ${ }^{12}$ the small differences are plausibly a result of the slight difference in the experimental temperatures, as the current data were obtained at $25{ }^{\circ} \mathrm{C}$, while the previously reported data were acquired at ambient temperature. The data indicate that hTDO exhibits similar affinity towards L-Trp and D-Trp, but the $k_{\text {cat }}$ towards L-Trp is much higher than D-Trp. The T342 mutation leads to the elevation of the $\mathrm{K}_{\mathrm{m}}$ to $1.19 \pm 0.09$ and $1.59 \pm 0.13 \mathrm{mM}$ for L-Trp and D-Trp, respectively (Fig. 5A); at the same time the $k_{\text {cat }}$ is reduced to $0.101 \pm 0.002$ and $0.082 \pm 0.002 \mathrm{~s}^{-1}$, respectively. As listed in Table 2, the T432A mutation results in similar reduction ( 10-fold) in the substrate affinity towards L-Trp and D-Trp, but it leads to much higher reduction in $k_{\text {cat }}$ for L-Trp ( 20-fold) as compared to D-Trp ( $\sim 3$-fold), indicating the abolishment of the substrate stereoselectivity (as manifested by the similar $k_{\mathrm{cat}} / \mathrm{K}_{\mathrm{m}}$ values of the mutant towards the two stereoisomers). Taken together the data confirm that T342 plays a pivotal role in controlling the substrate stereoselectivity of hTDO.

\section{Molecular dynamics simulations of the ternary complex of xcTDO}

To understand the molecular basis for the abolished substrate stereoselectivity in the T342A mutant of hTDO, we performed $20 \mathrm{~ns}$ MD simulations of the ternary complex of the T254A mutant of xcTDO (equivalent to T342A mutant of hTDO), in which $\mathrm{O}_{2}$ and Trp were bound to the active site. The data show that the structures of both the L- and D-Trp bound complexes remained stable during the $20 \mathrm{~ns}$ simulations, with a RMSD $<2.5 \AA$ with respect to the initial structure. (Figs. S5 and S6). In addition, the final averaged structures of the mutant show RMSD of 1.14 and $1.27 \AA$ with respect to the $\mathrm{L}$ and D-Trp-bound wild type enzyme, respectively, indicating that the mutation does not introduce global structural changes to the enzyme, consistent with the conclusion drawn from the aforementioned spectroscopic studies.

Visual inspection of the obtained MD trajectories, however, revealed important structural differences in the H-bonding network surrounding the $\mathrm{NH}_{3}{ }^{+}$group of the substrate. In the LTrp bound wild type enzyme, the substrate forms strong H-bonds with its surrounding environment, including (1) the indoleamine group and $\mathrm{H} 55$, (2) the $\mathrm{COO}^{-}$group and the sidechain groups of Y113/R117, and (3) the $\mathrm{NH}_{3}{ }^{+}$group, the OH group of T254, the propionate A group of the heme and the $\mathrm{OH}$ group of S124 (Fig. 6A). The replacement of LTrp with D-Trp leads to the interruption of the interaction between the $\mathrm{NH}_{3}{ }^{+}$group and 
T254, while the rest of the H-binding interactions remain almost the same. It is important to note that T254 and S124 are located in two loop regions that are held close together by an $\mathrm{H}$-bond between the $\mathrm{OH}$ group of T254 and the backbone carbonyl group of S123 (Fig. 6A). For clarity, the two loops are denoted as loop $250-260$ and loop $117-130$, respectively, hereafter.

In the L-Trp bound complex of the T254A mutant, the elimination of the H-bond between the $\mathrm{NH}_{3}{ }^{+}$group of the substrate and T254 leads to only subtle modifications in the Hbonding interactions with H55, Y113 and R117. However, it significantly increases the fluctuations of the loop $250-260$ (Fig. 6E), as evident in the much wider distance distribution of the A254-S123 pair (Fig. 6D). The opening of the two loops results in a dynamic distal pocket with fluctuating interactions between the $\mathrm{NH}_{3}{ }^{+}$group of the substrate and its surroundings. In addition, the $\mathrm{OH}$ group of $\mathrm{S} 124$ rotates to form an $\mathrm{H}$-bond with the $\mathrm{NH}_{3}{ }^{+}$ group, instead of the heme propionate A (Fig. 6B versus 6A). When the L-Trp is replaced by D-Trp in the mutant complex, the opening of the two loops was also observed (Fig. 6D), in addition, the heme propionate $\mathrm{A}$ forms an $\mathrm{H}$-bond with $\mathrm{S} 124$, instead of the $\mathrm{NH}_{3}{ }^{+}$group (Fig. 6C versus $6 \mathrm{~B}$ ).

\section{Molecular dynamics simulations of the ferryl/indole 2,3-epoxide intermediate of xcTDO}

To gain insight into the role of T254 in controlling catalysis, we performed $20 \mathrm{~ns}$ MD simulations of the recently characterized ferryl/indole 2,3-epoxide intermediate of the wild type xcTDO. ${ }^{14,27,28}$ The data show that both the complexes with the L- and D-isomers remained stable during the $20 \mathrm{~ns}$ simulation, showing a RMSD<2.5 ̊. (Figs. S7 and S8). In the previous studies we showed that, in the intermediate, the $\mathrm{NH}_{3}{ }^{+}$group forms a strong $\mathrm{H}-$ bond with the epoxide oxygen (Fig. 7A). This H-bond was demonstrated to be critical for triggering the ring opening reaction of the epoxide that ultimately leads to product formation. ${ }^{28,29}$ As observed in the ternary complex, in the epoxide intermediate of the wild type enzyme the $\mathrm{NH}_{3}{ }^{+}$group is held in position by H-bonding to the heme propionate $\mathrm{A}$ and T254; in addition, the loop $250-260$ and loop $117-130$ are held together by an H-bond between T254 and S123 (denoted as "closed" conformation hereafter) (Fig. 7A). When L-indole 2,3epoxide is replaced with the D-isomer, the H-bond between T254 and S123 is ruptured during the simulation, allowing the separation of the two loops (denoted as "open" conformation hereafter). Moreover, a spontaneous rotation of the $\mathrm{C}_{\alpha}-\mathrm{C}_{\beta}$ bond of the epoxide intermediate brings the $\mathrm{NH}_{3}{ }^{+}$group to a new position, thereby temporarily interrupting its $\mathrm{H}$-bonding interaction with the epoxide.

In the ferryl/L-indole 2,3-epoxide intermediate of T254A, the structure fluctuates between open and closed conformation (Fig. 7B) due to the absence of the H-bond between T254 and S123. When the L-indole 2,3-epoxide is replaced with the D-isomer only open conformation was observed. In the open conformation of both $\mathrm{L}$ - and $\mathrm{D}$-isomers, the $\mathrm{H}$-bond between the $\mathrm{NH}_{3}{ }^{+}$group and epoxide is broken, again due to the spontaneous rotation of the $\mathrm{C}_{\alpha}-\mathrm{C}_{\beta}$ bond. Additional calculations show that the estimated free energy barrier for the rotation of the $\mathrm{C}_{\alpha^{-}}$ $\mathrm{C}_{\beta}$ bond in the L-indole 2,3-epoxide intermediate of the wild type enzyme is very high ( 39 $\mathrm{kcal} / \mathrm{mol}$ ), as a result of steric hindrance (Fig. S9). The steric hindrance is significantly reduced $(\sim 3 \mathrm{kcal} / \mathrm{mol})$ for the $\mathrm{D}$-isomer, and becomes barrierless for both isomer complexes of the T254A mutant (Fig. S9). Finally, we performed additional $6 \mathrm{~ns}$ MD simulations of the open conformations of the L- and D-intermediate of the wild type and T254A mutant, without restraints. It was found that, in the L-indole 2,3-epoxide intermediate of the wild type enzyme, the $\mathrm{NH}_{3}{ }^{+}$group returned back to its initial position spontaneously; while in all other three cases, the $\mathrm{NH}_{3}{ }^{+}$group remained in the open conformation during the time scale of the simulations, confirming that the conversion of the close to open conformation removes the steric hindrance, thereby allowing the rotation of the $\mathrm{C}_{\alpha}-\mathrm{C}_{\beta}$ bond. 


\section{Mechanistic implications}

The simulation data of the ternary complex of xcTDO (with both L- and D-Trp) showed that

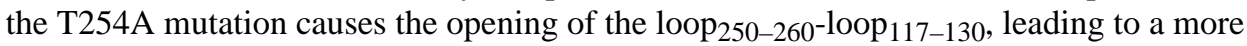
dynamic and open distal pocket, accounting for the much lower substrate affinity (i.e. higher $\mathrm{K}_{\mathrm{m}}$ ) observed in the activity studies (Table 2). On the other hand, the simulation data of the ferryl/L-indole 2,3-epoxide intermediate indicate that the T254A mutation also results in the opening of the loop $250-260^{-}$loop $_{117-130}$. It leads to local reorganization of the H-bonding interactions surrounding the $\mathrm{NH}_{3}{ }^{+}$group of the substrate, resulting in an open conformation, in which the $\mathrm{H}$-bond between the $\mathrm{NH}_{3}{ }^{+}$and the epoxide is temporarily lost. As the $\mathrm{H}$-bond is critical for catalyzing the ring opening reaction of the epoxide during the dioxygenase chemistry, ${ }^{28,} 29$ the data account for the $\sim 20$-fold reduction of the catalytic activity of the enzyme (see $k_{\text {cat }}$ in Table 2). Similar result was observed in the complex of the mutant with the D-isomer, accounting for the similar $k_{\text {cat }}$ value observed for the two stereoisomers (Table 2). Intriguingly, similar open conformation is observed for the wild type complex with the D-isomer, accounting for its much lower $k_{\text {cat }}$ with respect to the L-isomer, confirming that T254 plays a crucial role in determining the stereoselectivity of xcTDO.

\section{Conclusions}

In the previous work, we have revealed that hTDO exhibits similar affinities towards L- and D-Trp, but the $k_{\text {cat }}$ for L-Trp is 10 -fold higher with respect to D-Trp. ${ }^{12}$ The mutagenesis and MD simulation data obtained in this work demonstrate the critical role of T342 in hTDO (and the equivalent residue T254 in xcTDO) in controlling substrate binding, as well as substrate stereoselectivity of the enzyme, by modulating the H-bonding interaction between the $\mathrm{NH}_{3}{ }^{+}$group and epoxide oxygen of the ferryl/indole 2,3-epoxide intermediate of the enzyme, and by regulating the dynamics of the loop $_{250-260}$ and loop $117-130$. The crystallographic structural data show that substrate binding to xcTDO induces the closure/ organization of the two loops to sequester and stabilize the bound substrate. Sequence alignment along with the structural data of the substrate-free hIDO (the only structure available for hIDO) reveal that the substrate induced loop closure also occurs in hIDO, in addition, the T254 and S124 are fully conserved in hIDO, but S123 is replaced by a Gly.

It is plausible that, due to the absence of the T254-S123 interaction, hIDO displays $\sim 170$ fold higher affinity towards L-Trp with respect to D-Trp, but the $k_{\text {cat }}$ are similar for the two stereoisomers, ${ }^{12}$ opposite to what observed in hTDO. Apparently, T254 (T342) is not the only player in determining the substrate stereoselectivity of TDO; we are in a process of evaluating the role of S123 in the stereoselectivity of xcTDO. In any case, the mutagenesis results presented here agree perfectly with the prediction made in our previous work, in which T254 in XcTDO was revealed as one of the key residues for controlling the stereoselectivity of the enzyme. In this sense, our new experimental work validates the computational methodology, and confirms the predictive power of this tool.

\section{Supplementary Material}

Refer to Web version on PubMed Central for supplementary material.

\section{Acknowledgments}

Funding: This work was supported by National Institutes of Health Grant GM086482 and National Science Foundation Grant 1026788 to S.-R.Y, and National Institute of Health Molecular Biophysics Training Grant GM008572 to A.L.-B. It was also partially supported by grants from Universidad de Buenos Aires 08-X625 to MAM and 08-X074 to DAE, ANPCYT 07-1650 to MAM, and 06-25667 and Conicet PIP 01207 to DAE. DAE and MAM are members of CONICET. Computer power was gently provided by Cecar at FCEN, UBA and Cluster MCG at UNC. 
LC thanks CONICET, the Fulbright Foundation and the Bunge \& Born Foundation for the fellowships that allowed her to perform this work.

\section{Abbreviations}

$\begin{array}{ll}\text { TDO } & \text { Tryptophan dioxygenase } \\ \text { IDO } & \text { Indoleamine 2,3-dioxygenase } \\ \text { Trp } & \text { Tryptophan } \\ \text { hIDO } & \text { human IDO } \\ \text { NFK } & \text { N-formylkynurenine } \\ \text { hTDO } & \text { human TDO } \\ \text { rmTDO } & \text { Ralstonia metallidurans TDO } \\ \text { xcTDO } & \text { Xanthomonas campestris TDO } \\ \text { MD } & \text { Molecular Dynamics } \\ \text { 6C } & \text { six-coordinate } \\ \text { 5C } & \text { five-coordinate }\end{array}$

\section{References}

1. Sono M, Roach MP, Coulter ED, Dawson JH. Heme-Containing Oxygenases. Chem Rev. 1996; 96:2841-2888. [PubMed: 11848843]

2. Takikawa O. Biochemical and medical aspects of the indoleamine 2,3-dioxygenase-initiated Ltryptophan metabolism. Biochem Biophys Res Commun. 2005; 338:12-19. [PubMed: 16176799]

3. Knox WE, Mehler AH. The Conversion Of Tryptophan To Kynurenine In Liver: I. The Coupled Tryptophan Peroxidase-Oxidase System Forming Formylkynurenine. J Biol Chem. 1950; 187:419_ 430. [PubMed: 14794727]

4. Muller AJ, DuHadaway JB, Donover PS, Sutanto-Ward E, Prendergast GC. Inhibition of indoleamine 2,3-dioxygenase, an immunoregulatory target of the cancer suppression gene Bin1, potentiates cancer chemotherapy. Nature medicine. 2005; 11:312-319.

5. Munn DH, Shafizadeh E, Attwood JT, Bondarev I, Pashine A, Mellor AL. Inhibition of T Cell Proliferation by Macrophage Tryptophan Catabolism. The Journal of Experimental Medicine. 1999; 189:1363-1372. [PubMed: 10224276]

6. Grohmann U, Fallarino F, Puccetti P. Tolerance, DCs and tryptophan: much ado about IDO. Trends in Immunology. 2003; 24:242-248. [PubMed: 12738417]

7. Friberg M, Jennings R, Alsarraj M, Dessureault S, Cantor A, Extermann M, Mellor AL, Munn DH, Antonia SJ. Indoleamine 2,3-dioxygenase contributes to tumor cell evasion of T cell-mediated rejection. Int J Cancer. 2002; 101:151-155. [PubMed: 12209992]

8. Uyttenhove C, Pilotte L, Theate I, Stroobant V, Colau D, Parmentier N, Boon T, Van den Eynde BJ. Evidence for a tumoral immune resistance mechanism based on tryptophan degradation by indoleamine 2,3-dioxygenase. Nat Med. 2003; 9:1269-1274. [PubMed: 14502282]

9. Forouhar F, Anderson JLR, Mowat CG, Vorobiev SM, Hussain A, Abashidze M, Bruckmann C, Thackray SJ, Seetharaman J, Tucker T, Xiao R, Ma LC, Zhao L, Acton TB, Montelione GT, Chapman SK, Tong L. Molecular insights into substrate recognition and catalysis by tryptophan 2,3-dioxygenase. Proc Natl Acad Sci U S A. 2007; 104:473-478. [PubMed: 17197414]

10. Zhang Y, Kang SA, Mukherjee T, Bale S, Crane BR, Begley TP, Ealick SE. Crystal structure and mechanism of tryptophan 2,3-dioxygenase, a heme enzyme involved in tryptophan catabolism and in quinolinate biosynthesis. Biochemistry. 2007; 46:145-155. [PubMed: 17198384]

11. Sugimoto H, Oda S-i, Otsuki T, Hino T, Yoshida T, Shiro Y. Crystal structure of human indoleamine 2,3-dioxygenase: catalytic mechanism of $\mathrm{O} 2$ incorporation by a heme-containing dioxygenase. Proc Natl Acad Sci U S A. 2006; 103:2611-2616. [PubMed: 16477023] 
12. Batabyal D, Yeh SR. Human Tryptophan Dioxygenase: A Comparison to Indoleamine 2,3Dioxygenase. J Am Chem Soc. 2007; 129:15690-15701. [PubMed: 18027945]

13. Capece L, Lewis-Ballester A, Batabyal D, Di Russo N, Yeh SR, Estrin DA, Marti MA. The first step in the dioxygenation reaction carried out by Tryptophan dioxygenase and Indoleamine 2,3dioxygenase as revealed by QM-MM studies. J Biol Inorg Chem. 2010; 15:811-823. [PubMed: 20361220]

14. Capece, L.; Lewis-Ballester, A.; Yeh, S-R.; Estrin, DA.; Marti, MA. The complete Reaction Mechanism of Indoleamine 2,3- Dioxygenase as revealed by QM/MM simulations. 2011. Submitted

15. Lu C, Lin Y, Yeh SR. Inhibitory Substrate Binding Site of Human Indoleamine 2,3-Dioxygenase. J Am Chem Soc. 2009; 131:12866-12867. [PubMed: 19737010]

16. Capece L, Arrar M, Roitberg AE, Yeh SR, Marti MA, Estrin DA. Substrate stereo-specificity in tryptophan dioxygenase and indoleamine 2,3-dioxygenase. Proteins: Structure, Function, and Bioinformatics. 2010; 78:2961-2972.

17. Batabyal D, Yeh SR. Substrate-Protein Interaction in Human Tryptophan Dioxygenase: The Critical Role of H76. J Am Chem Soc. 2009; 131:3260-3270. [PubMed: 19209904]

18. Ishimura Y, Nozaki M, Hayaishi O, Nakamura T, Tamura M, Yamazaki I. The Oxygenated Form of 1-Tryptophan 2,3-Dioxygenase as Reaction Intermediate. J Biol Chem. 1970; 245:3593-3602. [PubMed: 5470825]

19. Egawa T, Yeh SR. Structural and functional properties of hemoglobins from unicellular organisms as revealed by resonance Raman spectroscopy. J Inorg Biochem. 2005; 99:72-96. [PubMed: 15598493]

20. Lewis-Ballester A, Batabyal D, Egawa T, Lu C, Lin Y, Marti MA, Capece L, Estrin DA, Yeh SR. Evidence for a Ferryl Intermediate in Heme-based Dioxygenases: Mechanistic Implications. Proc Natl Acad Sci U S A. 2009; 106:17371-17376. [PubMed: 19805032]

21. Berendsen HJC, Postma JPM, van Gunsteren WF, DiNola A, Haak JR. Molecular dynamics with coupling to an external bath. J Chem Phys. 1984; 81:3684-3690.

22. Wang J, Cieplak P, Kollman PA. How well does a restrained electrostatic potential (RESP) model perform in calculating conformational energies of organic and biological molecules? J Comput Chem. 2000; 21:1049-1074.

23. Marti MA, Crespo A, Capece L, Boechi L, Bikiel DE, Scherlis DA, Estrin DA. Dioxygen affinity in heme proteins investigated by computer simulation. J Inorg Biochem. 2006; 100:761-770. [PubMed: 16442625]

24. Bidon-Chanal A, Martí MA, Estrin DA, Luque FJ. Exploring the nitric oxide detoxification mechanism of mycobacterium tuberculosis truncated haemoglobin N. NATO Science for Peace and Security Series A: Chemistry and Biology. 2009:33-47.

25. Pearlman DA, Case DA, Caldwell JW, Ross WS, Cheatham TE, DeBolt S, Ferguson D, Seibel G, Kollman P. AMBER, a package of computer programs for applying molecular mechanics, normal mode analysis, molecular dynamics and. Comput Phys Comm. 1995; 91:1-41.

26. Marti MA, Capece L, Bidon-Chanal A, Crespo A, Guallar V, Luque FJ, Estrin DA. Nitric Oxide reactivity with Globins as investigated through computer simulation. Methods in enzymology. 2008; 437:477-498. [PubMed: 18433643]

27. Chung LW, Li X, Sugimoto H, Shiro Y, Morokuma K. Density functional theory study on a missing piece in understanding of heme chemistry: the reaction mechanism for indoleamine 2,3dioxygenase and tryptophan 2,3-dioxygenase. J Am Chem Soc. 2008; 130:12299-12309. [PubMed: 18712870]

28. Capece, L.; Lewis-Ballester, A.; Yeh, S-R.; Estrin, DA.; Marti, MA. The complete Reaction Mechanism of Indoleamine 2,3- Dioxygenase as revealed by QM/MM simulations. 2011. submitted

29. Chung LW, Li X, Sugimoto H, Shiro Y, Morokuma K. ONIOM Study on a Missing Piece in Our Understanding of Heme Chemistry: Bacterial Tryptophan 2,3-Dioxygenase with Dual Oxidants. J Am Chem Soc. 2010; 132:11993-12005. [PubMed: 20698527] 
(A)

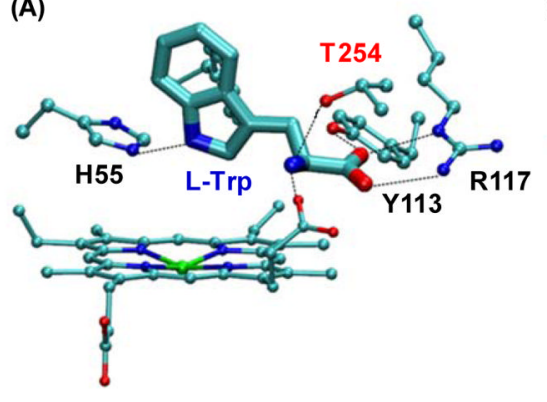

(B)

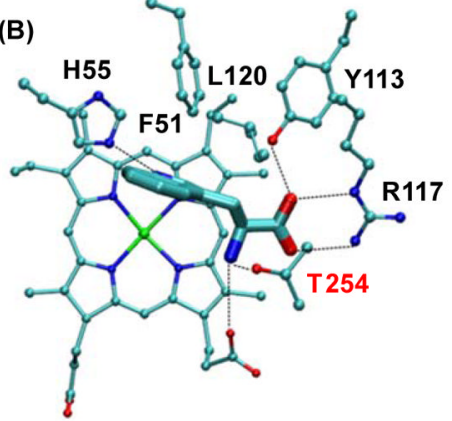

Figure 1.

Frontal (A) and top (B) views of the active site of L-Trp-bound xcTDO. ${ }^{9}$ The key residues stabilizing the bound L-Trp are highlighted and labelled in black and red. 

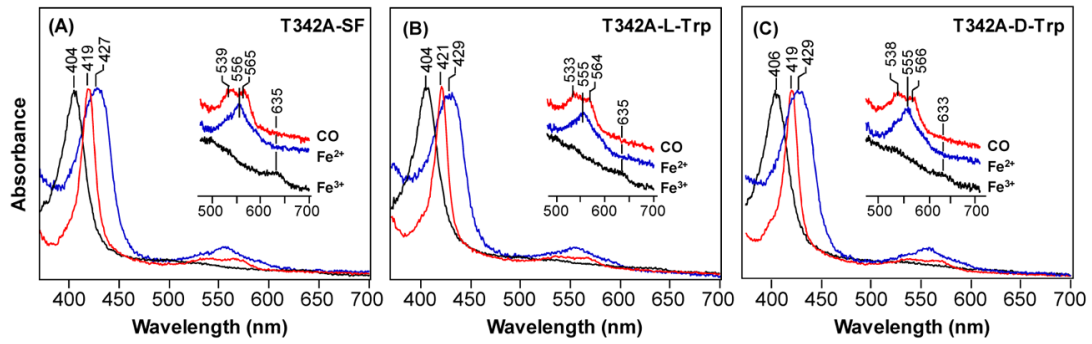

Figure 2.

Optical absorption spectra of the T342A mutant of hTDO in the substrate-free form (SF) (A), L-Trp-bound form (B) or D-Trp-bound form (C). Results for the ferric, ferrous and CObound forms are presented in black, blue and red, respectively. All the spectral intensities are normalized based on the Soret band. 


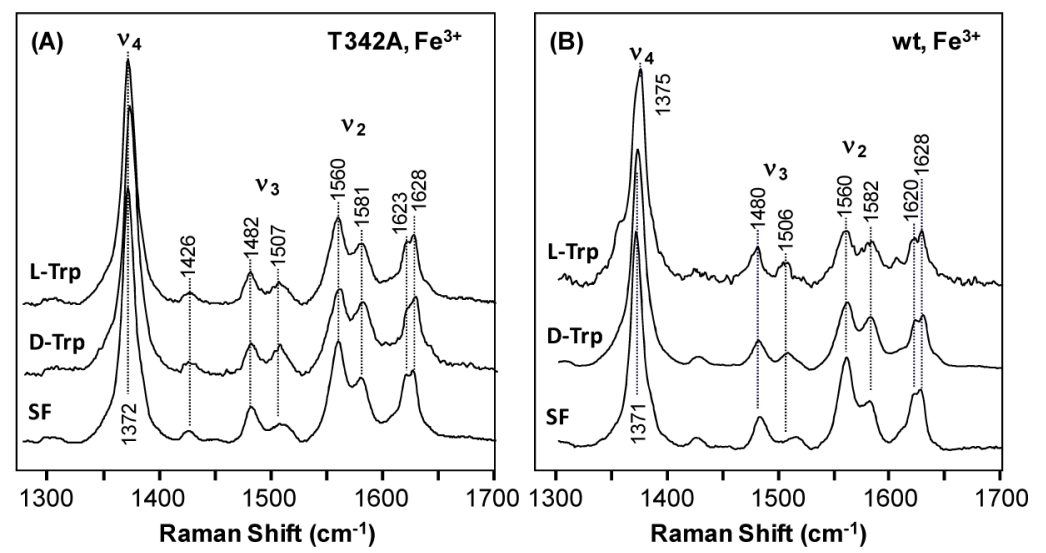

Figure 3.

Resonance Raman Spectra of the ferric derivative of the T342A mutant (A) and wild type (B) hTDO in the substrate-free (SF), L-Trp-bound form or D-Trp bound form. The spectra of the SF and L-Trp-bound form of the wild type enzyme are taken from literature. ${ }^{12}$ The Trp concentration used for generating the substrate-bound enzyme was $20 \mathrm{mM}$. 

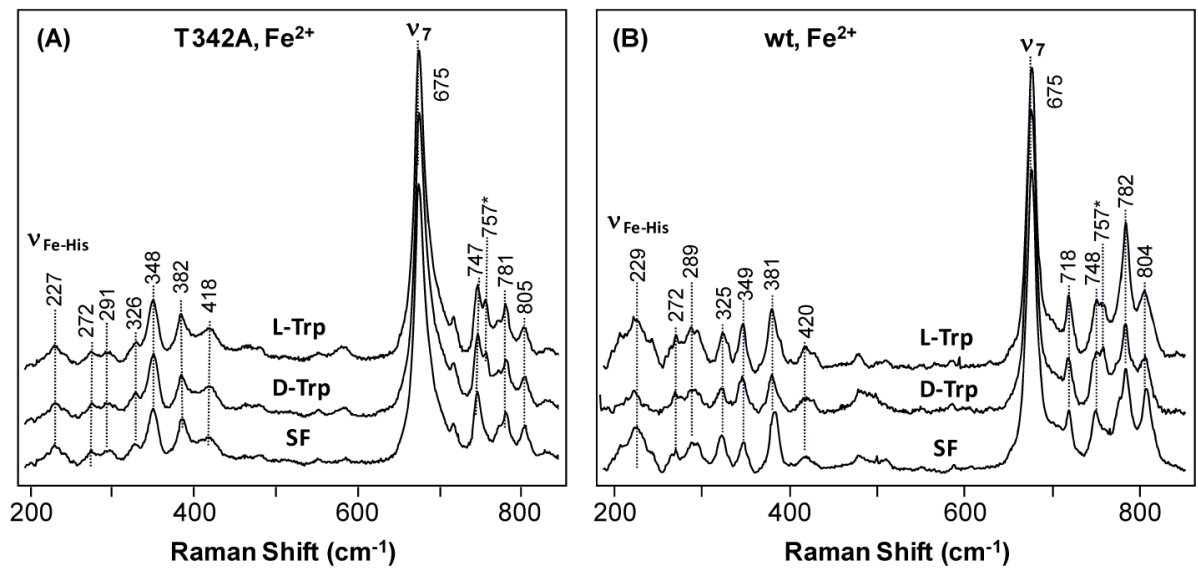

Figure 4.

Resonance Raman Spectra of the ferrous derivative of the T342A mutant (A) and wild type (B) hTDO, in the substrate-free (SF), L-Trp-bound form or D-Trp bound form. The spectra of the SF and L-Trp-bound form of the wild type enzyme are taken from literature. ${ }^{12}$ The Trp concentration used for generating the substrate-bound enzyme was $20 \mathrm{mM}$. The peak at $757 \mathrm{~cm}^{-1}$ indicated by an asterisk $(*)$ is derived from Trp substrate. 

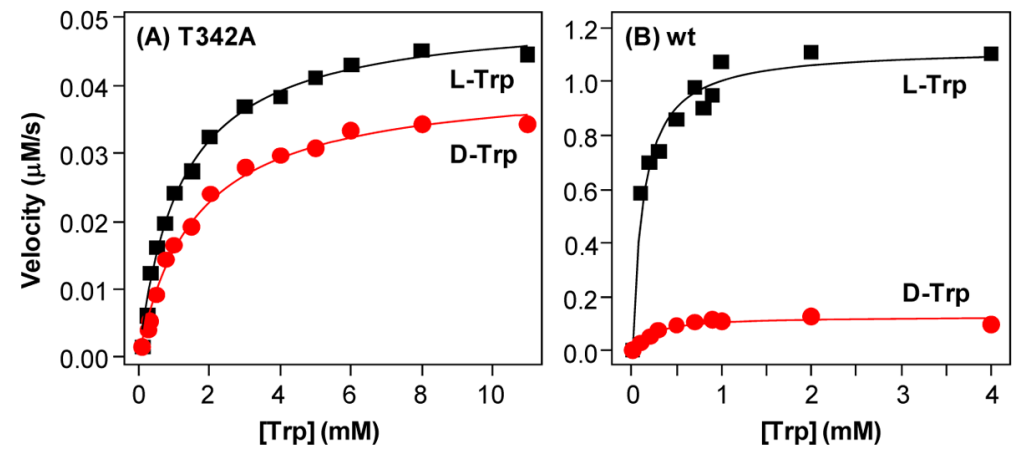

Figure 5.

Michaelis-Menten plots of the T342A mutant (A) and wild type (B) hTDO. The black and red symbols were obtained by using L-Trp and D-Trp as the substrate, respectively. The solid lines are best fit of the data with the Michaelis-Menten equation. The best fitted parameters are listed in Table 2. The enzyme concentration used for the measurements was $0.5 \mu \mathrm{M}$. 

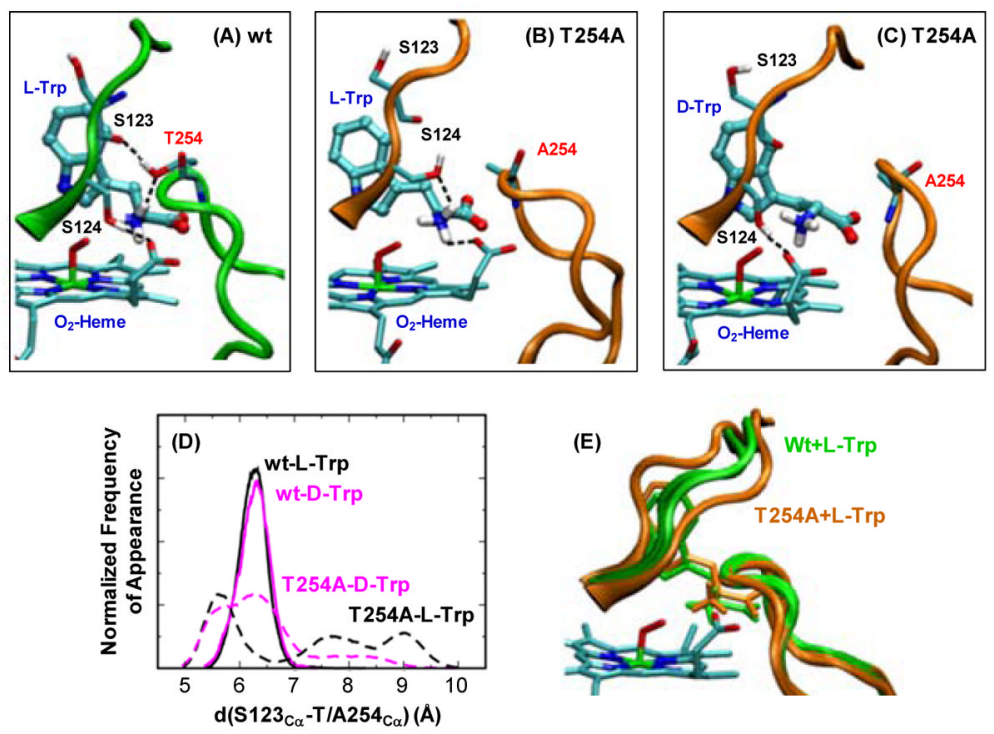

Figure 6.

Comparison of the active site structures of the ternary complex of the wild type (A) and T254A mutant (B-C) of xcTDO, obtained from MD simulations. Panel (D) shows the histogram of the loop $250-260-$ loop $_{117-130}$ separation, defined by the distance between the $\mathrm{C}_{\alpha}$ of S123 and the $\mathrm{C}_{\alpha}$ of T/A254, obtained from MD simulations. The data associated with Land D-Trp bound complexes are labelled in black and magenta, respectively; those associated with wild type and T254A are depicted in solid and dashed lines, respectively. Panel (E) shows superimposed two extreme structures of the ternary complex of the wild type (labelled in green) and T254A mutant (labelled in orange) of xcTDO, in which the

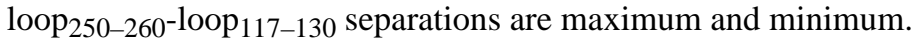



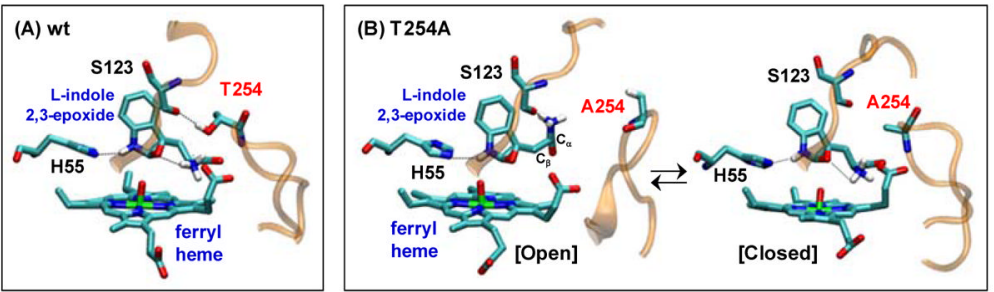

Figure 7.

Representative snapshots of the ferryl/L-indole 2,3-epoxide intermediate of the wild type (A) and T254A (B) of xcTDO obtained from MD simulations. 


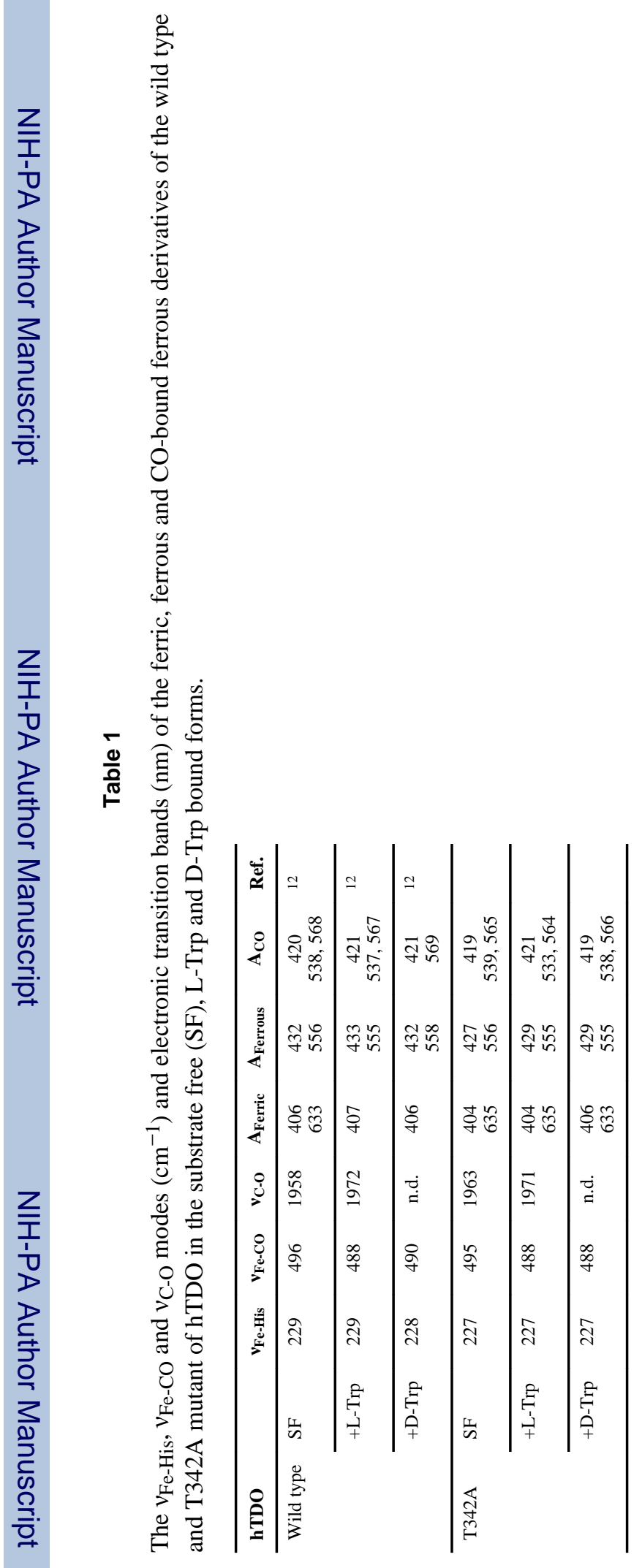


Table 2

Michaelis-Menten parameters of the wild type and T254 mutant of hTDO. All parameters are taken from the data presented in Figure 5.

\begin{tabular}{llccc}
\hline hTDO & Substrate & $\mathbf{K m}(\mathbf{m M})$ & $\boldsymbol{k}$ cat $\left(\mathbf{s}^{\mathbf{- 1}}\right)$ & $\boldsymbol{k}$ cat $/ \mathbf{K m}$ \\
\hline Wild type & L-Trp & $0.12 \pm 0.02$ & $2.24 \pm 0.08$ & 18.7 \\
& D-Trp & $0.20 \pm 0.06$ & $0.26 \pm 0.02$ & 1.3 \\
\hline T342A & L-Trp & $1.19 \pm 0.09$ & $0.101 \pm 0.002$ & 0.08 \\
& D-Trp & $1.59 \pm 0.13$ & $0.082 \pm 0.002$ & 0.05 \\
\hline
\end{tabular}

